\title{
Experiência Inicial no Fechamento Percutâneo da Comunicação Interatrial Tipo Ostium Secundum com a Prótese Figulla
}

\author{
Carlos A. C. Pedra ${ }^{1,2}$, Simone R. F. Pedra ${ }^{1,2}$, Rodrigo N. Costa ${ }^{1}$, Sérgio L. N. Braga ${ }^{1}$, \\ César A. Esteves ${ }^{1}$, Valmir F. Fontes ${ }^{1,2}$
}

\section{RESUMO}

Introdução: O tratamento percutâneo da comunicação interatrial tipo ostium secundum é a modalidade terapêutica de escolha em mais de $80 \%$ dos casos. Várias próteses encontram-se disponíveis para tal finalidade. Relatamos nossa experiência com a oclusão percutânea da comunicação interatrial com a prótese Figulla (Occlutech, Praga, República Tcheca), avaliando a segurança e a eficácia do método. Método: De abril de 2008 a março de 2010, foram realizados 25 procedimentos em dois centros de referência em 25 pacientes não-consecutivos (mediana de idade de 22 anos e de peso de $60 \mathrm{~kg}$ ) sob anestesia geral e monitoração pela ecocardiografia transesofágica. Do total de comunicações interatriais, 23 eram únicas, com diâmetro médio de $17,5 \pm 9,4 \mathrm{~mm}$, e 2 eram múltiplas. Em um paciente foi necessário o uso de duas próteses em duas comunicações interatriais distantes. O diâmetro médio das próteses foi de 20,8 $\pm 7,4 \mathrm{~mm}$, implantadas por bainhas longas de $9 \mathrm{~F}$ a $14 \mathrm{~F}$ pela veia femoral. Resultados: Houve sucesso no implante em todos os casos. Em um caso houve embolização logo após a liberação, com resgate do dispositivo da aorta descendente seguido de novo implante. O seguimento médio foi de 12 meses e o índice de oclusão foi de $100 \%$. Não houve complicações tardias. Conclusão: Nessa experiência inicial, a oclusão percutânea da comunicação interatrial com a prótese Figulla foi um procedimento de fácil execução, seguro e altamente eficaz.

DESCRITORES: Comunicação interatrial. Cateterismo cardíaco.

1 Hospital do Coração da Associação Sanatório Sírio - São Paulo, SP, Brasil.

2 Instituto Dante Pazzanese de Cardiologia - São Paulo, SP, Brasil. Correspondência: Carlos A. C. Pedra. Seção Médica de Intervenções em Cardiopatias Congênitas - Instituto Dante Pazzanese de Cardiologia. Av. Dr. Dante Pazzanese, 500 - 14ํandar - São Paulo, SP, Brasil - CEP 04012-180

E-mail: cacpedra@uol.com.br

Recebido em: 4/1/2010 • Aceito em: 4/3/2010

\section{ABSTRACT}

Initial Experience in the Percutaneous Occlusion of Ostium Secundum Atrial Septal Defect with the Figulla Device

Background: Percutaneous treatment of ostium secundum atrial septal defect is the preferred therapy in over $80 \%$ of cases. Several devices are available for this purpose. We report our experience with the percutaneous closure of atrial septal defect with the Figulla device (Occlutech, Prague, Czech Republic) assessing the safety and efficacy of the procedure. Method: From April 2008 to March 2010, 25 procedures were performed in 2 reference centers in 25 non-consecutive patients (median age 22 years and median weight $60 \mathrm{~kg}$ ) under general anesthesia and transesophageal echocardiographic monitoring. Atrial septal defects were single in 23 cases and multiple in 2 cases and had a mean diameter of $17.5 \pm 9.4 \mathrm{~mm}$. In one patient it was necessary to use two devices in two distant atrial septal defects. The mean diameter of the devices was $20.8 \pm 7.4 \mathrm{~mm}$ and they were implanted through 9-14 F long sheaths in the femoral vein. Results: Successful implantation was observed in all cases. In one case, there was embolization immediately after device release. The device was retrieved from the descending aorta followed by implantation of a new device. The mean follow-up was 12 months and the occlusion rate was $100 \%$. There were no late complications. Conclusion: In this initial experience, percutaneous occlusion of the atrial septal defect with the Figulla device was easy to perform, safe and highly effective.

KEY-WORDS: Heart septal defects, atrial. Heart catheterization.

A comunicação interatrial tipo ostium secundum é uma das cardiopatias congênitas mais frequentes, correspondendo a cerca de $10 \%$ de todas elas, sendo a mais comum em adultos. ${ }^{1,2} \mathrm{O}$ tratamento percutâneo das comunicações interatriais, especialmente com a prótese Amplatzer (AGA Medical, Minneapolis, Estados Unidos), vem sendo realizado com extrema segurança e eficácia na última década, tornando-se o 
Pedra CAC, et al. Experiência Inicial no Fechamento Percutâneo da Comunicação Interatrial Tipo Ostium Secundum com a Prótese Figulla. Rev Bras Cardiol Invasiva. 2010;18(1):81-8.

método terapêutico de eleição para tal doença na maior parte dos casos. ${ }^{3-11}$ Além da prótese Amplatzer, outras próteses foram introduzidas para uso clínico no Brasil, com a aprovação da Agência Nacional de Vigilância Sanitária (Anvisa), entre elas Figulla (Occlutech, Praga, República Tcheca), Helex (WL Gore, Flagstaff, Estados Unidos), Intrasept (Cárdia, Minnesota, Estados Unidos), Solysafe (Swissimplant, Solothurn, Suíça), Cera (LifeTech, Shenzhen, China) e Biostar (NMT, Boston, Estados Unidos). Cada uma dessas próteses apresenta vantagens e limitações, sendo fundamental, portanto, que se conheça melhor esses novos dispositivos para que se obtenham os melhores resultados. Nesse sentido, existe uma paucidade de informação na literatura em relação à prótese Figulla ASD Occluder (Occlutech). ${ }^{12-16}$ Neste artigo relatamos a experiência inicial no fechamento percutâneo da comunicação interatrial com essa prótese, avaliando a segurança e a eficácia do método.

\section{MÉTODO}

Trata-se de estudo prospectivo longitudinal observacional de uma coorte de pacientes submetidos a oclusão percutânea da comunicação interatrial tipo ostium secundum com a prótese Figulla ASD Occluder (Occlutech) de forma não-consecutiva em dois centros cardiológicos de referência. Os dados foram obtidos de forma retrospectiva. Os pacientes ou seus responsáveis legais foram adequadamente informados sobre os riscos e benefícios do procedimento e assinaram o termo de consentimento. O estudo foi aprovado pelo comitê de ética local.

\section{Critério de inclusão}

1. Crianças, adolescentes e adultos, de ambos os sexos, com diagnóstico clínico e ecocardiográfico de comunicação interatrial tipo ostium secundum (única ou múltiplas) com repercussão hemodinâmica, caracterizada pelo aumento do ventrículo direito com mais de dois desvios padrão da normalidade para a idade.

\section{Critérios de exclusão}

1. Comunicações interatriais de outros tipos.

2. Comunicações interatriais tipo ostium secundum com ventrículo direito de dimensões normais.

3. Doenças cardíacas associadas sem possibilidade de tratamento percutâneo concomitante, com necessidade de intervenção cirúrgica.

4. Doenças infecciosas ativas.

5. Alergia comprovada ao níquel.

6. Recusa em assinar o termo de consentimento.

7. Comunicação interatrial tipo ostium secundum de anatomia desfavorável para tratamento percutâneo, em decorrência da combinação de um ou mais fatores, incluindo grandes dimensões (maior que $35 \mathrm{~mm}$ no adulto); presença de mais de duas bordas contralaterais deficientes, finas e hipermóveis; proximidade das valvas atrioventriculares ou seio coronário; e necessidade de uso de próteses muito grandes para o tamanho do paciente, em especial crianças com peso inferior a $15 \mathrm{~kg}$. Tais fatores foram definidos por meio de ecocardiografia transesofágica antes do procedimento por profissional experiente na seleção de pacientes para tratamento percutâneo da comunicação interatrial. Não foram excluídos pacientes portadores de aneurisma do septo interatrial ou de apenas uma borda deficiente, qual fosse sua localização.

\section{Avaliação clínica pré-procedimento}

Os pacientes foram submetidos a avaliação clínica rotineira com obtenção de exames laboratoriais, incluindo eletrocardiograma, radiografia de tórax, ecocardiografia transtorácica, hemograma, coagulograma, eletrólitos, glicemia, avaliação da função renal, tipagem sanguínea e urina tipo I.

\section{Dispositivo}

A prótese Figulla ASD Occluder (Occlutech) é feita de malha de fios ultrafinos de nitinol (liga metálica de níquel e titânio), composta de dois discos conectados por cintura central de comprimento de $4 \mathrm{~mm}$ (Figuras 1 e 2) e de diâmetros de $6 \mathrm{~mm}$ a $39 \mathrm{~mm}$, com incrementos de $1,5 \mathrm{~mm}$ até $12 \mathrm{~mm}$ e de $3 \mathrm{~mm}$ entre $12 \mathrm{~mm}$ e $39 \mathrm{~mm}$. O diâmetro da cintura central determina o tamanho da prótese. Os discos direito e esquerdo, dependendo do tamanho do dispositivo, possuem diâmetros de $8 \mathrm{~mm}$ a $11 \mathrm{~mm}$ e de $12 \mathrm{~mm}$ a $15 \mathrm{~mm}$ maiores que a cintura, respectivamente. Na porção interna da malha de nitinol, encontram-se retalhos de poliéster que aumentam o poder de oclusão do dispositivo. ${ }^{12,13}$ Essa prótese é semelhante à prótese Amplatzer, possuindo, entretanto, algumas diferenças importantes. Os fios de nitinol são mais finos e numerosos, sendo individualmente entrelaçados. Além disso, estão

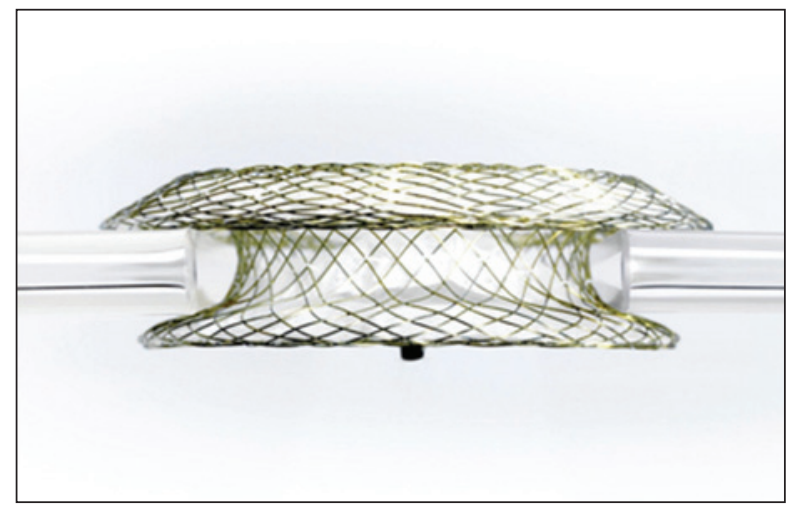

Figura 1 - Prótese Figulla tamanho $30 \mathrm{~mm}$. Nota-se a rede de nitinol formando dois discos conectados pela cintura central de $30 \mathrm{~mm}$. Há somente um pino de fixação dos fios da malha de nitinol, localizado no disco do lado direito. 
Pedra CAC, et al. Experiência Inicial no Fechamento Percutâneo da Comunicação Interatrial Tipo Ostium Secundum com a Prótese Figulla. Rev Bras Cardiol Invasiva. 2010;18(1):81-8.

fixados por mecanismo de solda a laser (e não clampeados como na Amplatzer) em apenas um ponto da prótese, localizado no pino fêmea proximal no disco do lado direito (Figura 3). Não há um ponto ou pino de fixação dos fios de nitinol no disco do lado esquerdo como na prótese Amplatzer, o que, teoricamente, otimiza a endotelização do dispositivo nesse lado ( Figuras 1 e 2). Além disso, tais características conferem maleabilidade e flexibilidade discretamente maiores em relação à Amplatzer. Por outro lado, por possuir mais fios de nitinol, são necessárias bainhas de perfil $1 \mathrm{~F}$ a 2 F maiores que a Amplatzer de mesmo tamanho para seu implante. Esse dispositivo também é autoexpansível e autocentrável (Figura 4) e é implantado por sistema que permite resgatá-lo e reposicioná-lo em qualquer momento até sua liberação final do cabo liberador, que se conecta ao pino fêmea proximal por mecanismo de rosca. Após ser enroscada no cabo liberador, deixamos uma mínima folga nesse mecanismo para que a prótese possa ser liberada com maior facilidade. Ela é carregada em introdutor curto de mesmo perfil

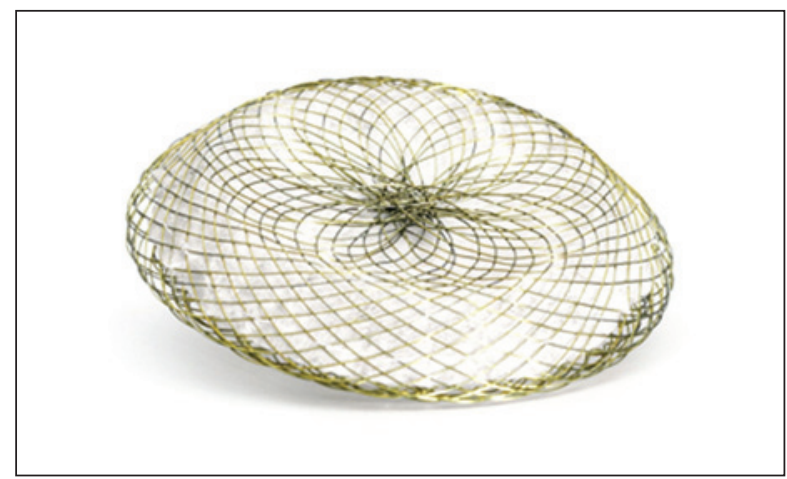

Figura 2 - Prótese Figulla vista do lado esquerdo. Não há pinos de fixação da malha no disco esquerdo. A porção interna da prótese é revestida por retalhos de poliéster.

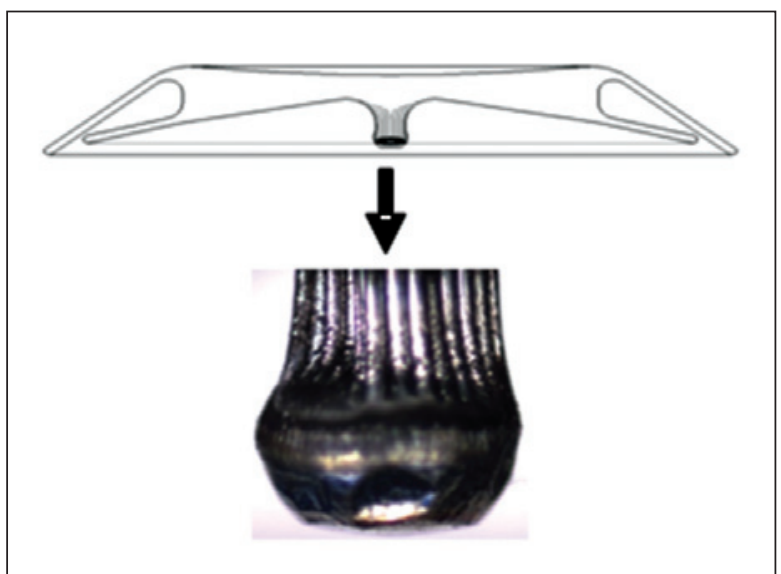

Figura 3 - Foto ampliada e desenho esquemático demonstrando o tipo de fixação dos fios de nitinol no pino do disco direito, utilizando um mecanismo de solda a laser, encontrado na Figulla. que a bainha longa. Tal prótese requer o uso de bainhas de $7 \mathrm{~F}$ a $14 \mathrm{~F}$ para o implante, dependendo do tamanho do dispositivo a ser utilizado. A prótese Figulla ASD Occluder possui o registro CE Mark desde março de 2007 e está liberada para uso clínico no Brasil com registro na Anvisa desde abril de 2008.

\section{Procedimento}

Depois de observado o período de jejum de oito horas, os pacientes realizaram cateterismo cardíaco direito e esquerdo rotineiro no laboratório de hemodinâmica sob anestesia geral e monitoração da ecocardiografia transesofágica (Figura 5). Depois de estabelecido o acesso venoso na veia femoral direita, sulfato de heparina foi administrado na dose de $100 \mathrm{UI} / \mathrm{kg}$ (máximo de $5.000 \mathrm{UI}$ ) juntamente com cefalotina $(30 \mathrm{mg} / \mathrm{kg}$, máximo de $1.000 \mathrm{mg}$ ). Angiografia na projeção hepatoclavicular foi obtida na maior parte dos casos. De forma semelhante a protocolos descritos anteriormente, ${ }^{3-7}$ a comunicação interatrial foi ultrapassada com

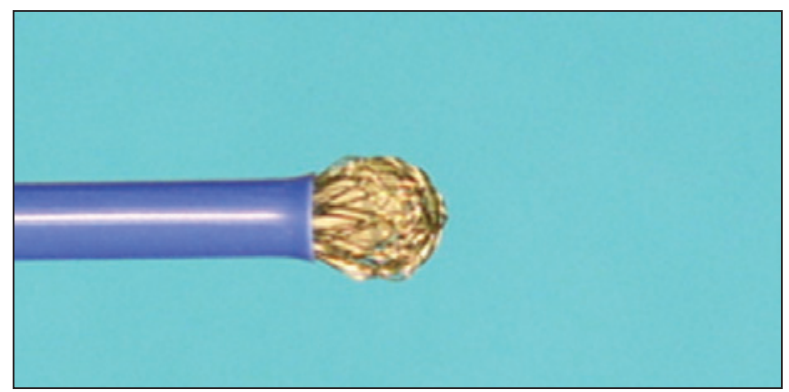

Figura 4 - Aspecto da prótese quando é empurrada para fora da bainha com a reconfiguração de seu desenho original. O dispositivo pode ser reposicionado e resgatado enquanto estiver conectado ao cabo liberador.

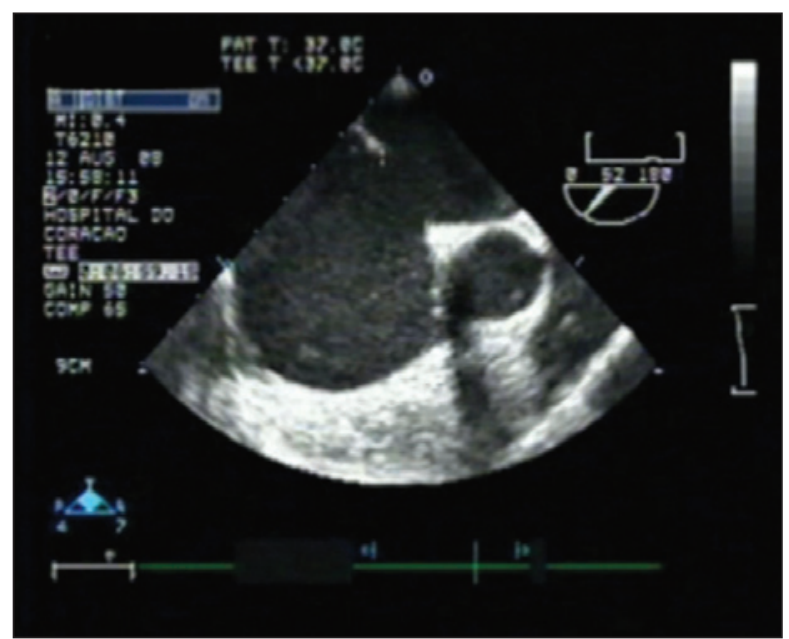

Figura 5 - Ecocardiografia transesofágica multiplanar (52 graus) em corte transversal pouco acima do nível da valva aórtica, demonstrando ampla comunicação interatrial com borda anterior deficiente. 
Pedra CAC, et al. Experiência Inicial no Fechamento Percutâneo da Comunicação Interatrial Tipo Ostium Secundum com a Prótese Figulla. Rev Bras Cardiol Invasiva. 2010;18(1):81-8.

um cateter Multipropósito ou Judkins de coronária direita e um guia rígido foi deixado na veia pulmonar superior esquerda. Nas comunicações interatriais com borda anterior deficiente $(<4-5 \mathrm{~mm})$ acompanhadas de septo posterior aneurismático ou fino e hipermóvel, associadas ou não a orifícios posteriores adicionais, foi determinada a medida do diâmetro estirado de forma semelhante a protocolos já publicados. Nesses casos foi escolhida uma prótese cuja cintura central era de $1 \mathrm{~mm}$ a $4 \mathrm{~mm}$ maior que o diâmetro estirado. Nas comunicações interatriais com bordas mais grossas ao seu redor, foi escolhida uma prótese cuja cintura central era de $20 \%$ a $30 \%$ maior que a maior medida do defeito pela ecocardiografia transesofágica, dispensando-se a realização do diâmetro estirado. Nos casos de defeitos múltiplos foi determinada a distância entre os vários defeitos de acordo com experiências prévias já publicadas. Em defeitos próximos, com distância menor que $7 \mathrm{~mm}$ a $8 \mathrm{~mm}$, a prótese foi implantada no maior defeito após realização do diâmetro estirado, com os discos cobrindo o defeito adicional, geralmente em localização posterior. Em defeitos com separação maior que $7 \mathrm{~mm}$ a $8 \mathrm{~mm}$, foram implantados dois dispositivos. Sobre o guia rígido, uma bainha longa tipo Mullins (Cook) com perfil apropriado para o tamanho da prótese escolhida ${ }^{7-14}$ foi avançada até o átrio esquerdo e a veia pulmonar superior esquerda pela comunicação interatrial. Após estabilização da bainha no átrio esquerdo, o guia e o dilatador foram retirados, assegurando-se a inexistência de ar no sistema. Um dispositivo de tamanho apropriado para o defeito foi avançado e implantado, seguindo as técnicas clássicas descritas para as próteses de nitinol. Nos casos de prolapso da porção anterior da prótese pela comunicação interatrial decorrente de rotação horária significativa da massa cardíaca secundária ao aumento do ventrículo direito (Figura 6), a bainha de Mullins foi rodada no sentido horário de forma a ficar mais paralela à coluna na fluoroscopia em projeção póstero-anterior (Figura 7). Dessa forma, o disco esquerdo adquiriu um ângulo mais paralelo ao septo interatrial (Figura 8), evitando o prolapso. No insucesso dessa técnica, outras medidas foram utilizadas, como abertura do disco esquerdo na veia pulmonar superior esquerda e uso de um segundo cateter ou de um cateter-balão para ancorar a porção anterior da prótese durante a abertura do disco direito.

O posicionamento final do dispositivo e a presença de fluxos residuais foram avaliados pela ecocardiografia. Confirmado o posicionamento correto, a prótese foi liberada e novas avaliações ecocardiográficas e radiológicas foram realizadas (Figuras 9 e 10).

\section{Avaliação pós-procedimento}

Após o procedimento, a hemostasia foi obtida por meio de compressão manual da região inguinal. Os pacientes foram acordados e extubados na sala de cateterismo, sendo transferidos para a unidade de re-

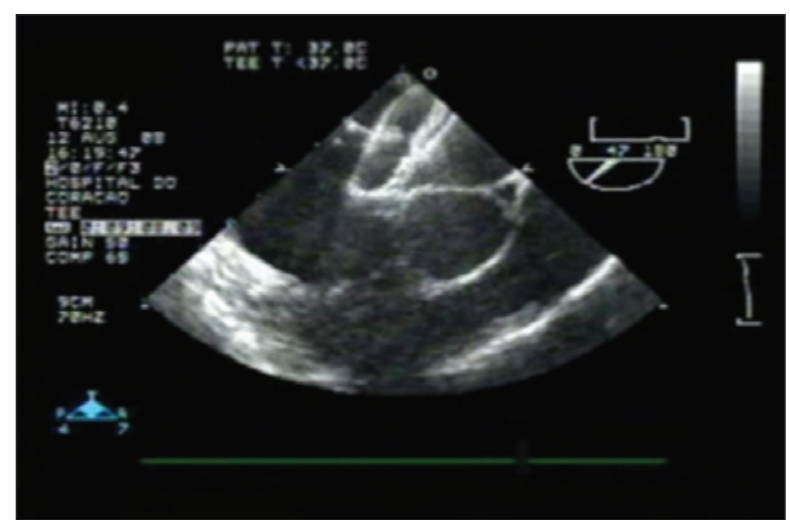

Figura 6 - Mesmo caso da Figura 5, ilustrando prolapso do disco esquerdo da prótese atrás da aorta.

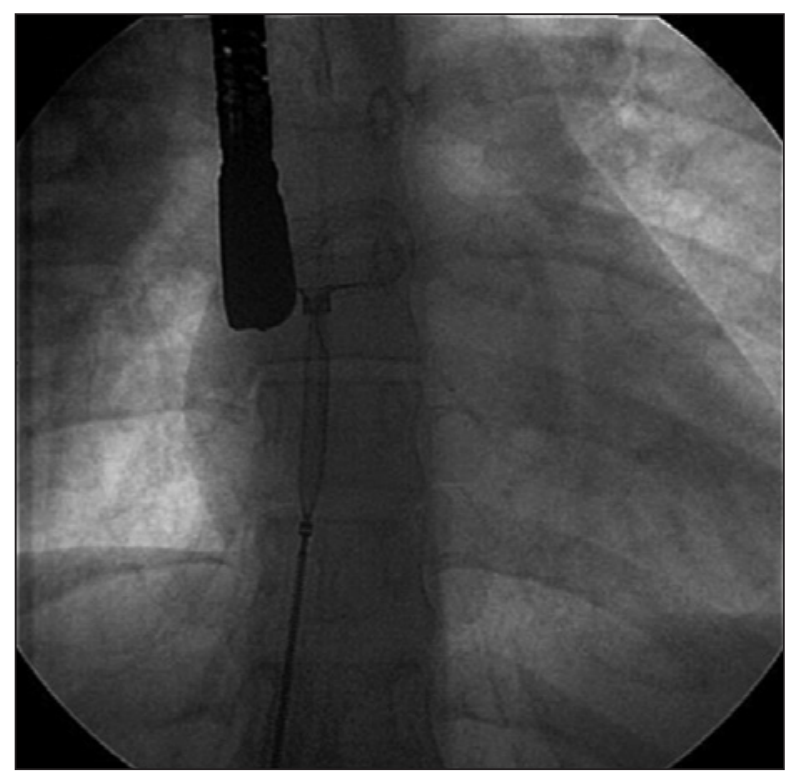

Figura 7 - Rotação horária da bainha com o cabo ficando em posição mais paralela à coluna.

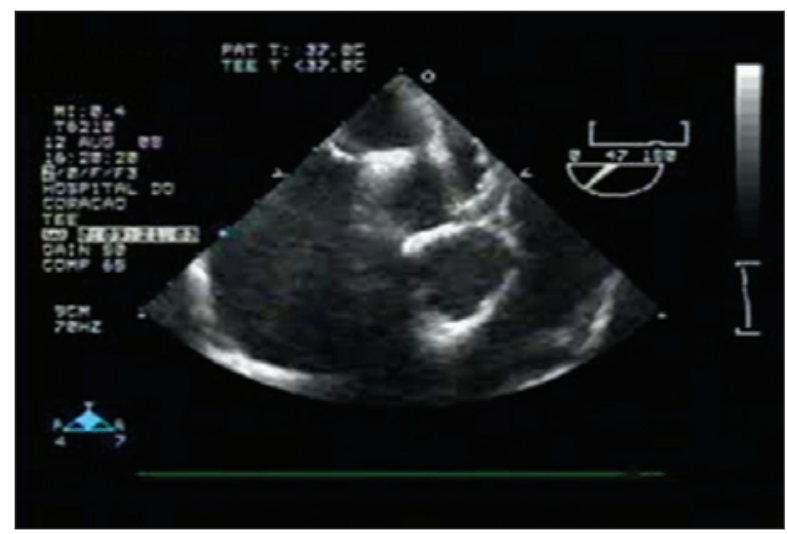

Figura 8 - Aspecto ecocardiográfico com o disco esquerdo vindo ao encontro do septo interatrial em ângulo mais paralelo e favorável após rotação horária da bainha longa. 
Pedra CAC, et al. Experiência Inicial no Fechamento Percutâneo da Comunicação Interatrial Tipo Ostium Secundum com a Prótese Figulla. Rev Bras Cardiol Invasiva. 2010;18(1):81-8.

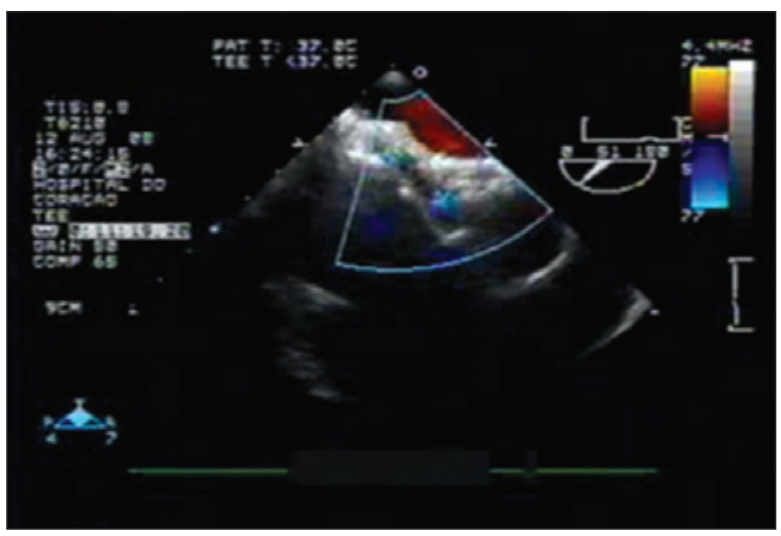

Figura 9 - Aspecto da prótese à ecocardiografia após a liberação do cabo. A parte anterior do dispositivo abraça a aorta. Há fluxo residual trivial pela malha do dispositivo.

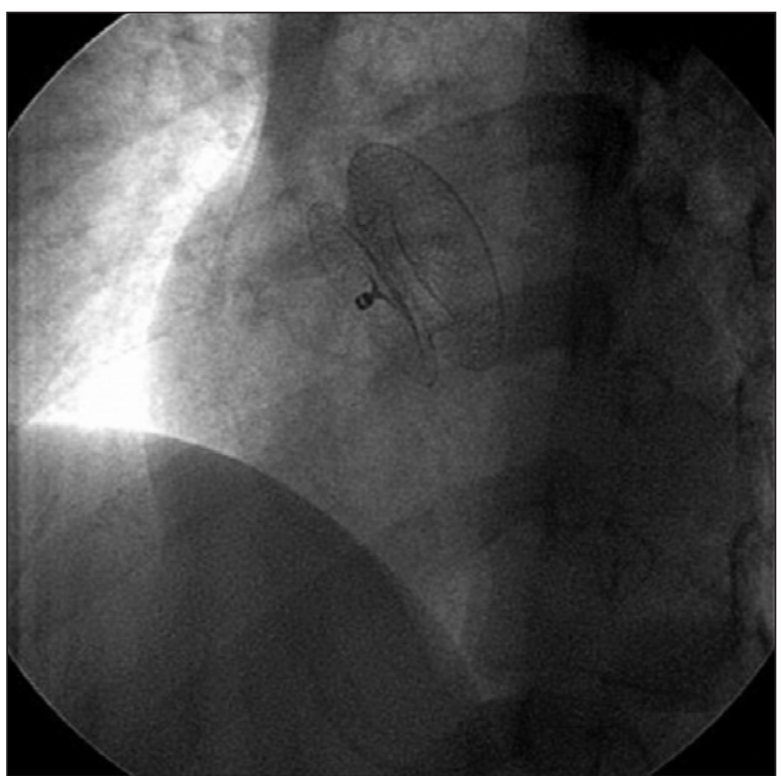

Figura 10 - Aspecto radiológico da prótese logo após sua liberação, demonstrando o pino fêmea no disco direito. O disco esquerdo não apresenta pinos de fixação dos fios de nitinol.

cuperação pós-anestésica para monitoração rotineira por cerca de uma a duas horas, para depois serem encaminhados ao quarto comum. No dia seguinte ao procedimento, foram submetidos a exame clínico rotineiro, com obtenção de exames laboratoriais, incluindo eletrocardiograma, radiografia de tórax e ecocardiografia transtorácica para avaliação do posicionamento da prótese e presença de possíveis fluxos residuais (Figura 11). Aqueles que estavam bem clinicamente e sem complicações receberam alta hospitalar, com orientação para tomar aspirina (dose de 3-5 mg/kg/dia) por seis meses e para seguir as recomendações clássicas para profilaxia de endocardite quando necessário nesse período.

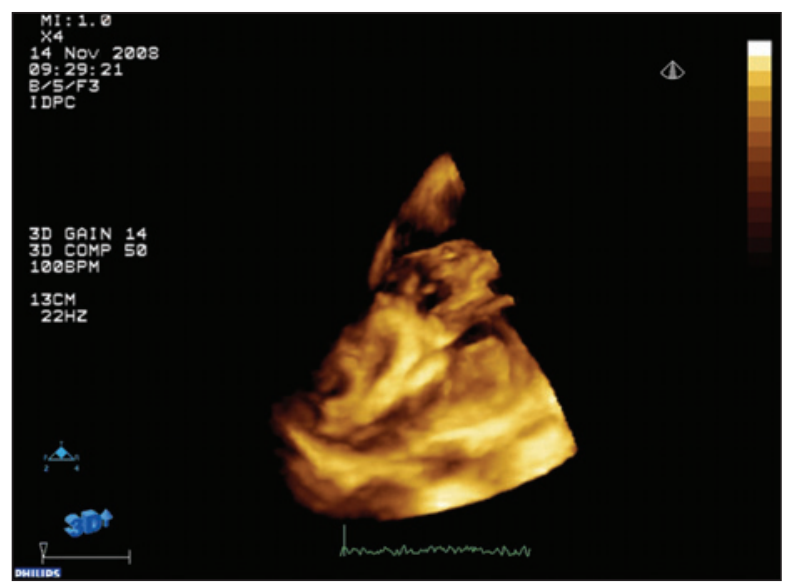

Figura 11 - Ecocardiograma transtorácico 3D em tempo real antes da alta hospitalar, demonstrando prótese vista do átrio direito em ótima posição e com baixo perfil.

O seguimento clínico constou de exame físico, realização de radiografia de tórax, eletrocardiograma e ecocardiografia transtorácica com Doppler em cores com 1, 3, 6 e 12 meses após o implante e a partir daí anualmente.

\section{Análise estatística}

Os valores são expressos em frequências e porcentagens, média e desvio padrão ou mediana e variação conforme distribuição da amostra.

\section{RESULTADOS}

\section{População e características dos defeitos}

De abril de 2008 a março de 2010, foram realizados 25 procedimentos em 25 pacientes. A mediana de idade foi de 22 anos (5,5 a 63 anos) e de peso, de $60 \mathrm{~kg}(18-86 \mathrm{~kg})$. Todos os pacientes apresentavamse bem clinicamente, em classe funcional I, controlados com pouca ou nenhuma medicação anticongestiva. Uma paciente de 18 anos era portadora de duas comunicações interatriais distantes, com separação de $14 \mathrm{~mm}$ entre elas. Um paciente era portador de duas comunicações interatriais próximas, com orifício adicional posterior pequeno de $3 \mathrm{~mm}$. Todos os pacientes restantes eram portadores de comunicações interatriais únicas. A média do maior diâmetro das comunicações interatriais foi de 17,5 $\pm 9,4 \mathrm{~mm}$, variando de $7 \mathrm{~mm}$ a $33 \mathrm{~mm}$. Alguma borda deficiente foi observada em 14 pacientes: em 12 pacientes a borda era retroaórtica, em 1 paciente a borda era póstero-superior (perto da veia cava superior) e em outro, póstero-inferior (perto da veia cava inferior). Em 5 casos o septo posterior era fino e redundante. Nos 17 casos nos quais foi realizado o diâmetro estirado, este foi de 22,1 $\pm 7,2 \mathrm{~mm}$. Todos os pacientes possuíam pressões normais ou discretamente elevadas na artéria pulmonar. 
Pedra CAC, et al. Experiência Inicial no Fechamento Percutâneo da Comunicação Interatrial Tipo Ostium Secundum com a Prótese Figulla. Rev Bras Cardiol Invasiva. 2010;18(1):81-8.

\section{Procedimentos}

Foram utilizadas 27 próteses nos 25 pacientes (tamanhos de $9 \mathrm{~mm}$ a $36 \mathrm{~mm}$; média de 20,8 \pm 7,4 mm). Como já foi relatado, uma paciente de 18 anos era portadora de duas comunicações interatriais distantes, sendo necessário o uso de dois dispositivos $(12 \mathrm{~mm}$ e $15 \mathrm{~mm}$ ). Na outra paciente com duas comunicações interatriais próximas, foi implantada apenas uma prótese (18 mm) na comunicação interatrial maior anterior, com cobertura adequada da comunicação interatrial menor posterior. Em todos os defeitos as próteses foram implantadas sem dificuldades técnicas. Entretanto, em 1 paciente de 25 anos com comunicação interatrial de $7 \mathrm{~mm}$ houve embolização de uma prótese de $9 \mathrm{~mm}$ para a aorta descendente logo após a liberação do cabo. O dispositivo foi resgatado pela via arterial, utilizando-se uma bainha $9 \mathrm{~F}$ e um cateter-laço que puxou o pino do disco direito sem dificuldades ou intercorrências. Esse paciente era portador de rede de Chiari hipertrofiada e o septo primum (lâmina da fossa oval) apresentava discreto desalinhamento com o septo secundum, com inserção na região retroaórtica do átrio esquerdo. Não havia sido realizado o diâmetro estirado nesse paciente. Após resgate, optou-se arbitrariamente pelo implante de uma prótese Figulla de oclusão de forame oval 27/30, realizado com sucesso. Em 2 casos tivemos dificuldades para desenroscar o cabo da prótese, sendo necessário recarregar o dispositivo em um caso e avançar a bainha até esta encostar na prótese no outro. Imediatamente após o implante, 7 pacientes apresentavam fluxos residuais triviais somente pela malha da prótese. O tempo médio de procedimento foi de 1,2 $\pm 0,5$ hora. Todos os pacientes tiveram alta no dia seguinte ao procedimento e não houve complicações intra-hospitalares.

\section{Seguimento}

O seguimento clínico variou de 3 meses a 22 meses, com mediana de um ano. A taxa de oclusão pela ecocardiografia foi de $100 \%$ (25/25). No seguimento, todos os pacientes encontravam-se em ritmo sinusal e apresentavam ventrículo direito com volume diastólico final de dimensões normais à ecocardiografia. Não houve episódios tardios de embolização, endocardite, arritmias, perfurações, fenômenos tromboembólicos ou disfunção das valvas atrioventriculares e semilunares.

\section{DISCUSSÃO}

Este artigo relata o uso clínico de mais uma prótese designada para a oclusão percutânea da comunicação interatrial tipo ostium secundum. Há pouca informação sobre a prótese Figulla na literatura, ${ }^{12-16}$ com alguns artigos relatando o uso da prótese para oclusão do forame oval. ${ }^{14,15}$ Por isso, achamos importante avaliar os resultados do uso desse novo dispositivo. Os desfechos observados comprovam a segurança e a eficá- cia da prótese Figulla ASD Occluder (Occlutech). É importante lembrar que tais resultados devem ser avaliados no contexto da experiência acumulada pelo nosso grupo nesse tipo de procedimento (> 400 casos), com uso de vários tipos de próteses em diversos tipos de anatomia. Postulamos que grupos com experiência já consolidada com a prótese Amplatzer no fechamento da comunicação interatrial não terão dificuldades para utilizar a prótese Figulla. Nesse sentido, os resultados do uso clínico de ambas as próteses são semelhantes nas mãos de um mesmo grupo. ${ }^{16}$ Por outro lado, especulamos que grupos com experiência pregressa limitada com o método podem apresentar curva de aprendizado mais lenta, com dificuldades técnicas e possíveis complicações.

Baseando-se nessa experiência prévia acumulada, modificamos ligeiramente alguns conceitos, diretrizes e técnicas que vinham sendo utilizadas nesse tipo de procedimento. O primeiro ponto refere-se à seleção dos pacientes. Antigos critérios de exclusão não são mais aplicados no contexto atual, ampliando o espectro de seleção de pacientes. A versatilidade de próteses como a Amplatzer e a Figulla permitiu a extensão desses antigos limites. Borda deficiente, bordas finas e hipermóveis, aneurismas do septo, defeitos múltiplos ou septo multifenestrado e amplos defeitos não constituem, isoladamente, critérios de exclusão. 6,11 Hoje em dia, estima-se que apenas cerca de $15 \%$ dos pacientes portadores de comunicação interatrial tipo ostium secundum não sejam candidatos ao tratamento percutâneo. ${ }^{9}$ Tais pacientes possuem combinação infrequente de fatores, incluindo defeitos muito amplos (> $35 \mathrm{~mm}$ no adulto) e mais de uma borda deficiente e fina ou aneurismática. O encontro de uma borda anterior (retroaórtica) deficiente é a regra nas comunicações interatriais (cerca de $40 \%$ a 50\%), principalmente nas maiores, e não deve ser encarada como contraindicação ao procedimento. ${ }^{4,11}$ Outra possível limitação para o método é a necessidade do uso de próteses demasiadamente grandes para o tamanho do paciente, especialmente em crianças $<15 \mathrm{~kg} .{ }^{9}$ De modo geral não utilizamos próteses Amplatzer maiores que $20 \mathrm{~mm}$ nesses pacientes. Nesse sentido, é importante medir o maior comprimento do septo interatrial em diversas projeções (a visão de 4 câmaras no ecocardiograma transtorácico pode ser muito útil) e lembrar que o disco do lado esquerdo da prótese $20 \mathrm{~mm}$ (Amplatzer ou Figulla) mede $34 \mathrm{~mm}$, devendo caber dentro do átrio esquerdo. Pensamos que as crianças pequenas com comunicação interatrial, em sua maioria, apresentam ganho ponderoestatural adequado e são totalmente assintomáticas, podendo tranquilamente esperar um período variado até atingir um peso mais apropriado e seguro para o procedimento (> 15-20 kg, $>$ 4-5 anos), sem comprometer desfechos de longo prazo, como o aparecimento de hipertensão pulmonar irreversível, arritmias e insuficiência cardíaca. Exceção a essa diretriz seria o encontro de pequenos 
Pedra CAC, et al. Experiência Inicial no Fechamento Percutâneo da Comunicação Interatrial Tipo Ostium Secundum com a Prótese Figulla. Rev Bras Cardiol Invasiva. 2010;18(1):81-8.

pacientes com pneumopatias crônicas graves (broncodisplasia da prematuridade, asmáticos perenes) muitas vezes portadores de quadros sindrômicos genéticos associados. ${ }^{17,18}$ Nesses subgrupos de risco, concordamos que o fechamento percutâneo oportuno da comunicação interatrial durante os dois primeiros anos de vida resulta em grande melhoria clínica e pulmonar. Por outro lado, próteses maiores vêm sendo utilizadas sem complicações significativas em crianças pequenas nos Estados Unidos e na Europa. ${ }^{18-20}$ Tais casos devem ser individualizados e discutidos com grupos com grande experiência com o método. Uma variação técnica que introduzimos nesta experiência foi a não realização do diâmetro estirado nos pacientes com comunicações interatriais de anatomia supostamente mais simples. Tal postura resultou em menor tempo de procedimento e menores custos. Por outro lado, o caso em que houve embolização do dispositivo para aorta descendente apresentava características que talvez requeiram a realização do diâmetro estirado, tais como mau alinhamento do septo primum com inserção no átrio esquerdo e hipertrofia da rede de Chiari. É possível que, se tivéssemos realizado o diâmetro estirado, teríamos obtido medidas significativamente maiores que $9 \mathrm{~mm}$, tamanho da prótese de comunicação interatrial utilizada. Ou seja: tal complicação provavelmente resultou mais de uma estratégia inadequada que do comportamento do dispositivo. Em decorrência da anatomia de base e do tamanho relativamente pequeno do defeito, foi possível sua oclusão com uma prótese de forame oval 27/30, de escoIha arbitrária. Além disso, acreditamos que em casos com borda anterior deficiente e septo posterior aneurismático ou mais fino e hipermóvel, especialmente quando há comunicação interatrial associada no septo posterior, a determinação do diâmetro estirado ainda é crucial para a escolha adequada do tamanho do dispositivo. Finalmente, nos casos de comunicações interatriais mais amplas, em que a porção anterior do disco esquerdo pode sofrer prolapso para o átrio direito, empregamos uma série de manobras para evitar tal ocorrência e viabilizar o implante. Destaque para a simples rotação horária da bainha, evitando-se o uso de bainhas com duas curvas (Hausdorf sheath), o que incrementaria os custos do procedimento.

Nesta experiência inicial, podemos observar algumas características vantajosas e desvantajosas no comportamento da prótese Figulla ASD Occluder. Essa prótese permite a oclusão de diferentes tamanhos de comunicações interatriais e com vasto espectro anatômico, conferindo versatilidade marcante ao dispositivo, de forma semelhante à observada com a prótese Amplatzer. Sua maior flexibilidade, ainda que discreta, resulta em melhor ângulo de ataque ao septo, principalmente em defeitos amplos com deficiência da borda anterior. Temos a impressão também que tal característica (maior maleabilidade) permite certo superdimensionamento em comunicações interatriais maiores sem comprometer a arquitetura do dispositivo, que abraça a região posterior da aorta. Se isso pode resultar em maior risco de erosão, ${ }^{21}$ só o acompanhamento de grande número de pacientes vai esclarecer essa questão. Até hoje não foram descritos na literatura casos de erosão com a prótese Figulla. Como os incrementos do tamanho da cintura da Figulla variam de $1,5 \mathrm{~mm}$ a $3 \mathrm{~mm}$, há menor quantidade de próteses por cada jogo ou kit completo. Tal característica reduz a necessidade de inventário com grande número de dispositivos e pode até auxiliar na escolha do dispositivo se considerarmos sua maleabilidade e a possibilidade de discreto superdimensionamento. Por outro lado, o maior número de fios de nitinol determina maior perfil do dispositivo, requerendo bainhas de maior calibre para o implante, o que pode limitar sua utilização em crianças pequenas. Por esse motivo, preferimos usar essa prótese em crianças maiores, adolescentes e adultos. $\mathrm{O}$ fato de termos encontrado dificuldades para desenroscar o cabo da prótese em dois casos denota a necessidade de deixar uma pequena folga no mecanismo de rosca para facilitar tal procedimento. Além disso, o cabo liberador tem certa rigidez, que exerce tensão no disco do lado direito antes da liberação final. Portanto, baseando-se nas observações apresentadas, acreditamos que ainda há espaço para melhorias nesse dispositivo e em seu sistema de liberação. A redução de seu perfil seria bem-vinda, especialmente para o uso em pacientes menores. A bainha poderia ser aramada, o que melhoraria seu torque, facilitando manobras de rotação nos casos de maior dificuldade técnica. O cabo liberador poderia ser composto de um fio de nitinol mais flexível em seus últimos $10 \mathrm{~cm}$ a $20 \mathrm{~cm}$, o que minimizaria a tensão no disco direito antes da liberação final. Nesse sentido, a empresa manufaturadora recentemente desenhou um novo mecanismo de conexão entre o cabo e o pino metálico do disco direito. Um sistema tipo biótomo foi introduzido, permitindo a rotação livre entre um pino esférico do disco direito e a mandíbula metálica da extremidade distal do cabo liberador. O registro de tal modificação ainda aguarda liberação pela Anvisa antes de ser introduzido na prática clínica. No caso observado de embolização, postulamos que a maior maleabilidade foi vantajosa para modificar a posição da prótese e puxá-la para dentro da bainha durante o resgate percutâneo pela via arterial. Por outro lado, a ausência do pino no lado esquerdo pode dificultar tais manobras ou mesmo impedir um possível resgate com um biótomo puxando o dispositivo pelo disco esquerdo.

\section{CONCLUSÃO}

Observou-se bom perfil de operabilidade, segurança e eficácia da prótese Figulla ASD Occluder nesta experiência inicial na oclusão percutânea da comunicação interatrial tipo ostium secundum. Constitui-se em outra boa alternativa no arsenal de próteses já disponíveis no mercado brasileiro. Obviamente, será 
Pedra CAC, et al. Experiência Inicial no Fechamento Percutâneo da Comunicação Interatrial Tipo Ostium Secundum com a Prótese Figulla. Rev Bras Cardiol Invasiva. 2010;18(1):81-8.

necessário grande número de pacientes, de preferência derivados de registros multicêntricos com monitoração independente, para melhor definir algumas questões importantes, como a reprodutibilidade de resultados em diferentes grupos, risco de erosão, fenômenos tromboembólicos ou outras possíveis complicações.

\section{CONFLITO DE INTERESSES}

Carlos A. C. Pedra é consultor técnico das seguintes empresas de representação de produtos médicos, participando de sessões de treinamento, casos ao vivo e palestras: Bioassist (Rio de Janeiro, RJ), TecMedic (Curitiba, PR), Allmed (São Paulo, SP), Neomex (Campinas, SP), Biomedical (Belo Horizonte, MG) e CMS Medical (São Paulo, SP), no Brasil; e AGA Medical Corporation (Minneapolis, Estados Unidos), WL Gore and Associates (Flagstaff, Estados Unidos), e Swissimplant (Solothurn, Suíça), no exterior.

Os demais autores declararam inexistência de conflito de interesses relacionado a este manuscrito.

\section{REFERÊNCIAS}

1. Carlgrem LE. The incidence of congenital heart disease in children born in Göteborg 1951-1960. Br Heart J. 1959;21: 40-50.

2. Rigby ML. Atrial septal defect. In: Gatzoulis MA, Webb GD, Daubeney PEF, editors. Diagnosis and management of adult congenital heart disease. London: Churchill Livingstone; 2003. p.163-70.

3. Fontes VF, Pedra CAC, Pedra SRF, Esteves CA, Braga SLN, Assef JE, et al. Experiência inicial no fechamento percutâneo da comunicação interatrial com a prótese Amplatzer. Arq Bras Cardiol. 1998;70:147-53.

4. Braga SLN, Sousa AGMR, Pedra CAC, Esteves CA, Pedra SRFF, Fontes VF. Efetividade clínica e segurança do tratamento percutâneo da comunicação interatrial tipo ostium secundum com a prótese Amplatzer. Arq Bras Cardiol. 2004; 83(Espl):7-13.

5. Pedra CAC, Pedra SRF, Fontes VF. Atrial septal defects of secundum type. From surgical to percutaneous treatment and the dinossaurs of the future. Arq Bras Cardiol. 2003; 80:650-5.

6. Pedra CAC, Pedra SRFF, Esteves CA, Cassar R, Pontes Jr SC, Braga SLN, et al. Percutaneous closure of secundum atrial septal defects with complex anatomy. J Invasive Cardiol. 2004;16:117-22.

7. Neves JR, Arrieta SR, Cassar RS, Pedra SRFF, Braga SLN, Esteves CA, et al. Tratamento percutâneo versus cirúrgico da comunicação interatrial tipo ostium secundum em adultos. Rev Bras Cardiol Invasiva. 2006;14:126-32.

8. Butera G, Carminati $M$, Chessa $M$, Youssef $R$, Drago $M$, Giamberti A, et al. Percutaneous versus surgical closure of secundum atrial septal defect: comparison of early results and complications. Am Heart J. 2006;151:228-34.

9. Butera G, Romagnoli E, Carminati M, Chessa M, Piazza L, Negura D, et al. Treatment of isolated secundum atrial septal defects: impact of age and defect morphology in 1,013 consecutive patients. Am Heart J. 2008;156:706-12.

10. Du ZD, Hijazi ZM, Kleinman CS, Silverman NH, Larntz K; Amplatzer Investigators. Comparison between transcatheter and surgical closure of secundum atrial septal defect in children and adults: results of a multicenter nonrandomized trial. J Am Coll Cardiol. 2002;39:1836-44.

11. Du ZD, Koenig P, Cao QL, Waight D, Heitschmidt M, Hijazi ZM. Comparison of transcatheter closure of secundum atrial septal defect using the Amplatzer septal occluder associated with deficient versus sufficient rims. Am J Cardiol. 2002;90:865-9.

12. Halabi A, Hijazi ZM. A new device to close secundum atrial septal defects: first clinical use to close multiple defects in a child. Catheter Cardiovasc Interv. 2008;71:853-6.

13. Peruga J, Krecki R, Chrzanowski Ł, Krzemiñska-Pakuła $M$, Kasprzak JD. Implantation of the Figulla Occlutech device in a patient with an ostium secundum atrial septal defect. Kardiol Pol. 2008;66:658-60.

14. Krizanic F, Sievert H, Pfeiffer D, Konorza T, Ferrari M, Figulla HR. Clinical evaluation of a novel occluder device (Occlutech) for percutaneous transcatheter closure of patent foramen ovale (PFO). Clin Res Cardiol. 2008;97:872-7.

15. Krecki R, Peruga JZ, Plewka L, Krzemiñska-Pakuła M, Kasprzak JD. Implantation of a Occlutech Figulla PFO occluder in a patient with patent foramen ovale and history of embolic stroke. Cardiol J. 2008;15(4):380-2.

16. Pac A, Polat TB, Cetin I, Oflaz MB, Balli S. Figulla ASD occluder versus Amplatzer septal occluder: a comparative study on validation of a novel device for percutaneous closure of atrial septal defects. J Interv Cardiol. 2009;22:489-95.

17. Pedra SRF, Jatene MB, Pedra CAC. Hybrid management of a large atrial septal defect and a patent ductus arteriosus in an infant with chronic lung disease. Ann Pediatr Cardiol. 2010. In press.

18. Diab KA, Cao QL, Bacha EA, Hijazi ZM. Device closure of atrial septal defects with the Amplatzer septal occluder: safety and outcome in infants. J Thorac Cardiovasc Surg. 2007;134:960-6.

19. Vogel M, Berger F, Dähnert I, Ewert P, Lange PE. Treatment of atrial septal defects in symptomatic children aged less than 2 years of age using the Amplatzer septal occluder. Cardiol Young. 2000;10:534-7.

20. Butera G, De Rosa G, Chessa M, Rosti L, Negura DG, Luciane $\mathrm{P}$, et al. Transcatheter closure of atrial septal defect in young children: results and follow-up. J Am Coll Cardiol. 2003;42:241-5.

21. Amin Z, Hijazi ZM, Bass JL, Cheatam JP, Hellenbrand WE, Kleinnam CS, et al. Erosion of Amplatzer septal occluder device after closure of secundum atrial septal defects: review of registry of complications and recommendations to minimize future risk. Catheter Cardiovasc Interv. 2004;63:496-502. 